\title{
Evaluation of antibody response to BNT162b2 mRNA COVID-19 vaccine in patients affected by immune-mediated inflammatory diseases up to 5 months after vaccination
}

\author{
Davide Firinu $^{1}$ - Andrea Perra ${ }^{2,3} \cdot$ Marcello Campagna $^{1} \cdot$ Roberto Littera $^{3,4} \cdot$ Giuseppe Fenu $^{5} \cdot$ Federico Meloni $^{1}$. \\ Selene Cipri ${ }^{4}$. Francesca Sedda ${ }^{2}$. Maria Conti ${ }^{1}$ - Michela Miglianti ${ }^{1}$. Giulia Costanzo ${ }^{1}$. Marta Secci ${ }^{1}$. \\ Gianmario Usai ${ }^{1} \cdot$ Mauro Giovanni Carta $^{1} \cdot$ Riccardo Cappai $^{6}$. Germano Orrù ${ }^{1}$. Stefano Del Giacco ${ }^{1}$. \\ Ferdinando Coghe $^{6} \cdot$ Luchino Chessa $^{1,3}$
}

Received: 4 August 2021 / Accepted: 27 October 2021 / Published online: 5 November 2021

(c) The Author(s) 2021

\begin{abstract}
SARS-CoV-2 vaccination with mRNA product BNT162b2 elicited high immunogenicity in healthy subjects in trials. This study aims to better understand the factors that influence the humoral immune response to vaccination against SARS-CoV-2 in patients with immune-mediated inflammatory diseases (IMIDs). We enrolled patients and healthy healthcare workers control group (HCW) that underwent mRNA BNT162b2 vaccination and measured the serum IgG anti-S-RBD response at booster dose (T1), one month after booster dose (T2) and up to 5 months (T3). Demographic, disease-specific and vaccination data were recorded. Vaccination response of 551 participants naïve to SARS-CoV-2 infection were included in HCW and 102 in the IMID group, analyzing separately those on anti-CD20. At T2 all naïve HCW developed anti-S-RBD-IgG, while $94 \%$ of IMID responded $(p<0.001)$. IMID patients had a significantly different level of IgG than HCW at both T1 $(p=0.031), \mathrm{T} 2$ $(p<0.001)$, while there was no significant difference at $\mathrm{T} 3$. There were no statistically significant differences according to the IMID type or to ongoing treatment with immunosuppressants, corticosteroids or biological drugs other than anti-CD20. The proportion and magnitude of response was significantly lower in IMID treated with anti-CD20 drugs. There was a correlation with age at T1 and at T2 but not at T3, stronger in patients than in HCW. Immune response close after BNT162b2 vaccination is reduced in patients with IMID, but there is no significant difference at 5 months. The measured reduction is related to age and the disease itself rather than treatments, with the exception of anti-CD20 drugs.
\end{abstract}

Keywords Covid-19 $\cdot$ Vaccination $\cdot$ Immunogenicity $\cdot$ Autoimmune disease $\cdot$ IMID $\cdot$ BNT162b2

Davide Firinu

davide.firinu@unica.it

1 Department of Medical Sciences and Public Health, Policlinico Universitario - AOU di Cagliari, University of Cagliari, Azienda Ospedaliero Universitaria, SS 554-Bivio Sestu, 09042 Monserrato, Cagliari, CA, Italy

2 Department of Biomedical Sciences, University of Cagliari, Cagliari, Italy

3 Associazione per l'Avanzamento della Ricerca per i Trapianti O.d.V., Non Profit Organisation, Cagliari, Italy

4 Medical Genetics, Department of Medical Sciences and Public Health, University of Cagliari, Cagliari, Italy

5 Department of Neuroscience, A.O. Brotzu, Cagliari, Italy

6 Laboratory Clinical Chemical Analysis and Microbiology, University Hospital of Cagliari, Cagliari, Italy

\author{
Abbreviations \\ CLIA Chemiluminescent analytical system \\ COVID-19 \\ Coronavirus disease 2019 \\ csDMARDs Conventional synthetic and targeted syn- \\ thetic DMARDs \\ IMID Immune-mediated inflammatory diseases \\ HCW Healthy healthcare workers \\ PRNT Plaque reduction neutralization test \\ RBD Receptor binding domain
}

SARS-CoV-2 Severe acute respiratory syndrome coronavirus 2 


\section{Introduction}

The extent of the profound immunological and non-immunological responses linked to SARS-CoV-2 infection is currently being investigated worldwide, due to the huge death-toll of SARS-CoV-2 pandemic and the short-term consequences of COVID-19. The first SARS-CoV-2 vaccines are among the most remarkable science and medicine accomplishments in modern history and offer realistic hope for an end to the COVID-19 pandemic.

Epidemiological models to estimate the magnitude and timing of future COVID-19 cases, given different assumptions regarding the protective efficacy and duration of the adaptive immune response to SARS-CoV-2, range widely from sustained epidemics to near elimination [1]. Even in the best-case scenario of an apparent elimination, a resurgence in contagion could be possible as late as 2024 [2]. Thus, to alleviate the direct consequences of the ongoing pandemic detailed and reliable data on the epidemiology, natural history and treatment possibilities of SARS-CoV-2 infection, as well as about vaccine response are needed. Vaccination represents the only reliable means to quickly mitigate the spread and impact of COVID-19 in the forthcoming period. Assessment and post-vaccine monitoring of anti-SARS-CoV-2 antibody specifically targeting and thereby inactivating the spike protein and/or its receptor binding domain (RBD) may inform about the humoral response and its duration [3].

The Pfizer-BioNTech COVID-19 (BNT162b2) vaccine consisting of a lipid nanoparticle-formulated mRNA vaccine encoding the prefusion spike glycoprotein of SARS-CoV-2 received the conditional marketing authorization from the European Medicine Agency (EMA) on December 21, 2020 and was approved by the Food and Drug Administration (FDA) on January 14, 2021. The overall immune response to BNT162b2 has been reported [4], inducing SARS-CoV-2 specific spike-protein (and/or its RBD) B-cells and neutralizing antibody response and generation of specific polyfunctional CD $8+$ and CD4 + T-cells. Although the overall immune effects of the BNT162 b2 vaccine has been reported, the profile of humoral immune profile remain to be further investigated in selected subgroups such as immune-mediated inflammatory diseases (IMID) [5].

Since infections are a relevant cause of morbidity and mortality in patients with IMID [6], it is relevant to address this question because the worldwide spread of the SARS-CoV-2 infection forces a rapid vaccination of patients suffering from these diseases.

Moreover, in these patients the infection risk may be even higher for both the altered regulation of the immune system itself and for the immunosuppressive effects of medications $[7,8]$.
Indeed, vaccinations in this population are complicated by disease-modifying immunosuppressive agents or antirheumatic drugs (either conventional, targeted synthetic or biologicals), which modulate or suppress key targets of the immune system and potentially decrease the immunogenicity and efficacy of the vaccines [9].

However, there is scant available data on real-world cohorts of vaccinated subjects and about response to COVID-19 mRNA vaccines in patients affected by IMID [10-14], in particular beyond 4-8 weeks after the full vaccination course. In a prospective observational study focused on COVID-19 infection and vaccination (CORIMUN study), we tested the antibody response to SARS-CoV-2 spike protein over 5 months after vaccination. This study aimed to describe the characteristics of humoral response induced by mRNA-based vaccine BNT162b2 [15] in subjects affected by IMID and analyze the impact of treatments.

\section{Methods}

\section{Patient selection}

We enrolled consecutive subjects aged $>18$ years, having received COVID-19 vaccine Pfizer/BioNTech BNT162b2 (two doses 21-days apart of $30 \mu \mathrm{g}$ mRNA vaccine Comirnaty by Pfizer Inc, NY, USA) and deliberately given their Informed Consent to participate in the study. Pregnancy, transplantation, known immunodeficiency or lymphoproliferative disorders were exclusion criteria. Among enrolled subjects, we defined two groups:

(a) Vaccinated subjects at our hospital with concurrent IMID, grouped as: (1) ankylosing spondylitis (AS), psoriasis, psoriatic arthritis (PsA); (2) rheumatoid arthritis (RA) (3) systemic lupus erythematosus; (4) miscellaneous systemic disorders (5) inflammatory bowel disease (6) multiple sclerosis;

(b) A control group of well-characterized subjects enrolled among healthy healthcare workers (HCW) enrolled at our hospital, without any of the immune-mediated diseases listed above, no evidence of immunodeficiency or taking relevant medications.

To ascertain prior infection with SARS-CoV-2, subjects were asked if they had a positive PCR test in the past and were cross-matched with the database of positive rt-PCR tests at the laboratory and hospital records. We also checked data of serological testing for health surveillance in HCW from June to December 2020 of both IgM and IgG antibodies with the 2019-nCov (Snibe, Shenzhen, China) chemiluminescent analytical system (CLIA) assay on MAGLUMI platform that detects antibodies of natural infection to 
SARS-CoV-2 Spike-(S) protein and N-protein with high sensitivity and specificity.

Each group included both naïve and previously infected (rt-PCR confirmed infection) subjects. Naïve subjects had not been previously infected by SARS-CoV-2 (testing since April 2020 gave repeatedly negative rt-PCR test for SARS$\mathrm{CoV}-2$; repeatedly undetectable $\operatorname{IgG}$ or IgM antibodies, also at the end of December 2020, before vaccination).

\section{Sample collection and storing}

$10 \mathrm{~mL}$ of peripheral blood was obtained by venipuncture immediately before each vaccine dose and defined as T0, before first dose; $\mathrm{T} 1$, at second vaccine shot $(+21$ days from $\mathrm{T} 0)$; $\mathrm{T} 2$ at day $51(\mathrm{~T} 1+28$ days); $\mathrm{T} 3$ at day 151 after the first vaccine dose. The serum was separated by centrifugation $(2000 \times \mathrm{g}$ for $15 \mathrm{~min})$ within $3 \mathrm{~h}$ of collection and aliquots were stored at $-80^{\circ} \mathrm{C}$ until use.

\section{Serological studies}

The antibody response induced by vaccine to $S$ protein (primary endpoint) was detected with the anti-SARS-CoV-2 S-RBD IgG (Snibe Diagnostics, New Industries Biomedical Engineering Co., Ltd, Shenzhen, China) on a MAGLUMI analyzer, to titrate levels of specific IgG at specific timepoints. Analytical and clinical features of the assay, including the correlation with neutralization by using plaque reduction neutralization test (PRNT) 50 titer has been previously investigated [16].

\section{Statistical analysis}

Patient characteristics were summarized using means, medians, standard deviations, ranges and percentages as appropriate. Chi-squared tests of independence and Fischer's exact tests were used for categorical data. Mann-Whitney $U$ and Kruskal-Wallis tests were used for unpaired continuous data. Linear regression was used to evaluate the relationship between the dependent variable (antibody titer) and the clinical and demographic characteristics of patients as independent variables. All reported p-values represent 2-tailed tests, with $p \leq 0.05$ considered statistically significant. All variables were analyzed using SPSS.

\section{Ethical aspects}

Patients were recruited and enrolled in the study protocol at the University Hospital of Cagliari. Written informed consent was obtained from all patients and controls in accordance with the ethical standards (institutional and national) of the local human research committee. The study protocol, including informed consent procedures, conforms to the ethical guidelines of the Declaration of Helsinki and was approved by the responsible ethics committee (Ethics Committee of the Cagliari University Hospital approval May 27, 2020; protocol number GT/2020/10894 and extension approved January 27, 2021). Records of written informed consent are kept on file and are included in the clinical record of each patient.

\section{Results}

Among subjects vaccinated and naïve to SARS-CoV-2 Infection, 551 participants were included in the control group (HCW), and 102 were in the IMID group. Vaccinated HCW with previous SARS-CoV-2 infection were 111, and 9 in the IMID group. Baseline characteristics of both groups are shown in Table 1. Mean study dropout rate at T3 versus T2 was of $15 \%$.

Both cohorts included only Caucasian participants, and patients continued their treatment before or after the two vaccine doses, without stopping or tapering their drug(s), only avoiding the administration of injective drugs on \pm 3 days of the vaccine date.

Due to the well-known impairment of immunogenicity linked to anti-CD20 agents [17], patients taking this medication were analyzed separately.

There was no difference in age, gender, BMI and cigarette smoking comparing these groups (Kruskall-Wallis $\chi^{2}$, $p>0.05)$.

\section{Immunogenicity of BNT162b2 in SARS-CoV-2 naïve patients with IMID and controls}

All subjects in the naive HCW group developed a positive antibody response (defined as $>1 \mathrm{AU} / \mathrm{ml}$ or higher) at $\mathrm{T} 1$ (364/364) and T2 (551/551) as compared with 90\% (27/30) at $\mathrm{T} 1$ and $94 \%$ (63/67) at T2 in the IMID group. A statistically significant difference was found at $\mathrm{T} 1(p=0.002)$ and T2 ( $p<0.001$; Fisher's exact test).

At $\mathrm{T} 1$, the median IgG anti-S-RBD levels in the IMID group were $14.08 \mathrm{AU} / \mathrm{mL}$ (IQR, 5.08-27.8), increasing to 146.39 AU/mL (IQR, 53.93-295) at T2 and then decreasing to $60.65 \mathrm{AU} / \mathrm{mL}$ (IQR, 21.1-96,55) at T3 (-59\% vs. T2).

In HCW controls, at T1 the median IgG anti-S-RBD levels were 23.66 AU/mL (IQR, 9.76-47.9), increasing to 217 $\mathrm{AU} / \mathrm{mL}$ (IQR, 134.8-389.9) at T2 and then decreasing to 46.76 AU/mL (IQR, 26.26-81.57) at T3 (-79\% vs. T2).

The median IgG anti-S-RBD levels in the IMID group were significantly lower than control group test at $\mathrm{T} 1$ $(p=0.031)$ and $\mathrm{T} 2(p=0,000,233)$, while there was no significant difference at T3 $(p=0.172)$ (Fig. 1). 
Table 1 Characteristics of patients and controls

\begin{tabular}{|c|c|c|c|c|c|}
\hline Subjects characteristics & $\begin{array}{l}\mathrm{HCW}, \mathrm{SARS}-\mathrm{CoV}-2 \\
\text { naive } \\
(n=551)\end{array}$ & $\begin{array}{l}\text { IMID, } \\
\text { SARS-CoV-2 naive } \\
(n=95)\end{array}$ & $\begin{array}{l}\text { IMID treated with } \\
\text { anti-CD20 naive } \\
(n=7)\end{array}$ & $\begin{array}{l}\text { HCW, previous } \\
\text { SARS-CoV-2 } \\
(n=111)\end{array}$ & $\begin{array}{l}\text { IMID } \\
\text { previous SARS-CoV-2 } \\
(n=9)\end{array}$ \\
\hline Age (years), median & $51(39-58)$ & $56(42.5-66)$ & $58(50-69.25)$ & $48(35-55)$ & $50(30.5-61.5)$ \\
\hline Males, $\%$ & 31.8 & 27.5 & 14.3 & 38.7 & 33.3 \\
\hline BMI (IQR) & $23.18(20.79-25.95)$ & $23.56(21.4-26.6)$ & $21.77(19.9-23.7)$ & $23.93(21.66-26.32)$ & $22.65(20.47-25.53)$ \\
\hline Current smoker, $\%$ & 16.6 & 16.9 & 14.3 & 11.5 & 11 \\
\hline Diabetes, $\%$ & 3.3 & 6.4 & 14.3 & 3.8 & 11.1 \\
\hline \multicolumn{6}{|l|}{ IMID type } \\
\hline SLE & 0 & $19(23.5)$ & $2(28.6)$ & 0 & $1(11.1)$ \\
\hline RA & 0 & $19(23.5)$ & $1(14.3)$ & 0 & $2(22.2)$ \\
\hline PsA, Psoriasis, AS & 0 & $20(24.7 \%)$ & & 0 & $1(11.1)$ \\
\hline $\begin{array}{l}\text { Miscellaneous systemic } \\
\text { disorders }^{\mathrm{a}}\end{array}$ & 0 & $16(19.8)$ & $2(28.6)$ & 0 & $1(11.1)$ \\
\hline IBD & 0 & $4(4.9)$ & & 0 & $2(22.2)$ \\
\hline Multiple sclerosis & 0 & $3(3.7)$ & $2(28.6)$ & 0 & $2(22.2)$ \\
\hline \multicolumn{6}{|c|}{ Immunomodulatory treatment } \\
\hline Glucocorticoids, $n(\%)$ & 0 & $43(53.1)$ & $5(71.4)$ & 0 & $2(22.2)$ \\
\hline Prednisone eq/day, mg & 0 & $5(5-8.75)$ & $5(5-10)$ & 0 & $3.75(2.5-3.75)$ \\
\hline $\begin{array}{l}\text { Glucocorticoid and/or } \\
\text { any immunosuppressive } \\
\text { drug, } n(\%)\end{array}$ & $0(0)$ & $70(74.5)$ & $6(87.5)$ & $0(0)$ & $5(55.6)$ \\
\hline Anti-TNF- $\alpha n(\%)$ & $0(0)$ & $11(13.6)$ & $0(0)$ & $0(0)$ & $1(11.1)$ \\
\hline $\begin{array}{l}\text { Non-anti-TNF biologicals } \\
\text { (ustekinumab, secuki- } \\
\text { numab, abatacept, } \\
\text { tocilizumab and vedoli- } \\
\text { zumab) } n(\%)\end{array}$ & $0(0)$ & $10(12.3)$ & $0(0)$ & $0(0)$ & $0(0)$ \\
\hline $\operatorname{csDMARDs}^{\mathrm{b}} n(\%)$ & $0(0)$ & $41(51.2)$ & $3(42.9)$ & $0(0)$ & $1(11.1)$ \\
\hline Other drugs ${ }^{\mathrm{c}} n(\%)$ & $0(0)$ & $5(6.3)$ & $0(0)$ & $0(0)$ & $1(11.1)$ \\
\hline
\end{tabular}

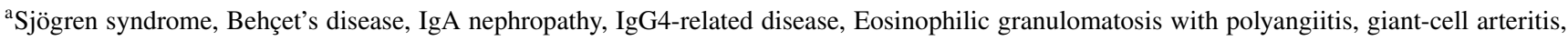
autoimmune hepatitis and UCTD

b csDMARDs: methotrexate, sulfasalazine, leflunomide, hydroxychloroquine, mycophenolate, azathioprine and cyclosporin

${ }^{\mathrm{c}}$ Interferon, colchicine, JAK-inhibitors, belimumab and dimethyl fumarate

\section{IMID subgroups and antibody levels}

There were no statistically significant differences between subgroups of IMID enrolled in the study for age, BMI and proportion of patients currently treated with immunosuppressive treatment (Kruskal-Wallis test).

The rate of seropositivity at $\mathrm{T} 2$ was similar among the subgroups of diseases included in the study and there were no statistically significant differences between subgroups of subjects at T1, T2 and T3 $(p>0.05$, Kruskal-Wallis test).

The median IgG anti-S-RBD levels and IQR at T1 and T2 for each IMID subgroup are presented in the supplementary materials, Table S1.

\section{Correlation of treatments and antibody levels}

Comparing the response to vaccination in patients treated with GC, GC and/or immunosuppressive drug we did not find a significant difference in IgG anti-S-RBD levels at T1, T2 and T3 $(p>0,05)$. Similarly, we found no differences for those under anti-TNF-alpha drugs (median of those treated at T2 139.7 AU/mL; IQR, 61-279) for the subgroups of RA and PsA/Psoriasis, or for those treated with biologicals different from TNF-alpha inhibitors (ustekinumab, secukinumab, abatacept, tocilizumab and vedolizumab) $(p>0,05)$, csDMARDs (supplementary materials, Table S2), or for other drugs (interferon, 


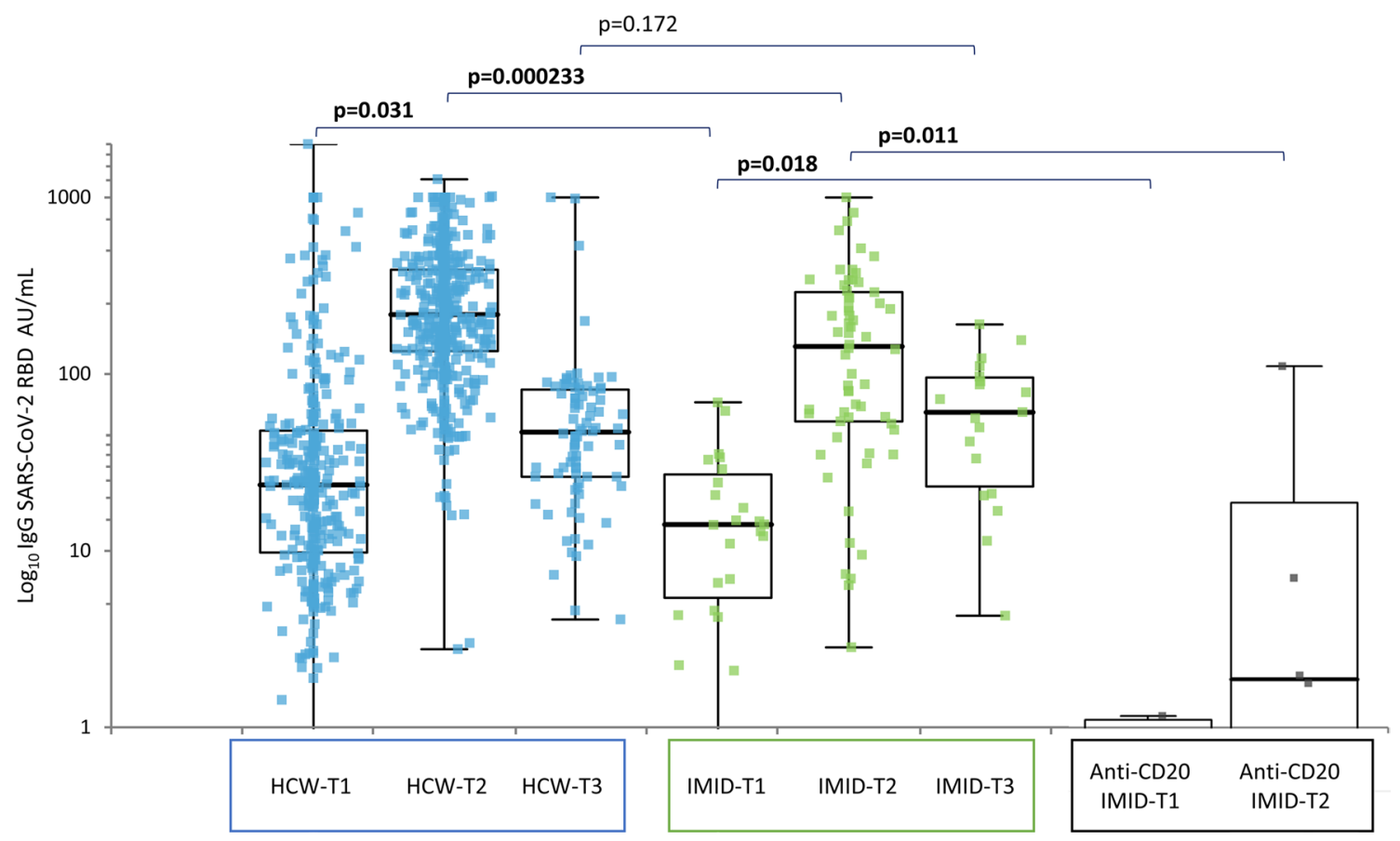

Fig. 1 SARS-CoV-2 anti-S-RBD IgG immune responses in naïve patients and control subjects. IgG titer anti-S-RBD IgG (AU/L) elicited by BNT162b2 at T1 (booster dose), T2 (28 days after booster) and T3 (151 days after first dose) among healthy healthcare workers

colchicine, Jak-inhibitors, belimumab and dimethyl fumarate); (for all at T1, T2 T3; comparison drug vs. no drug).

\section{Correlation with subjects' characteristics}

Among the demographic characteristics, age could influence the response to vaccination in both patients and healthy controls. In fact, the Spearman test showed a correlation between age and antibody response at $\mathrm{T} 1$ and at $\mathrm{T} 2$. This correlation is stronger in patients than in healthy controls (T1: rho: -0.24 in HCW vs. -0.41 in patients). At T3, there was no correlation between age and antibody response (supplementary materials, Tables S4 and S5).

Linear regression models were tested including IgG antiRBD titer at each timepoint (T1, T2 and T3) as dependent variable and age, gender, glucocorticoid, treatment with immunosuppressant, treatment with biologicals, disease duration as independent variables. No significant relationship between IgG titer at T1, T2 and T3 and multiple combinations of clinical and demographic variables was detected except for age (supplementary materials, Table S6).
$(\mathrm{HCW})$ and IMID subjects and IMID treated with anti-CD20 agents (anti-CD20 IMID), naïve to SARS-CoV-2 infection. All subjects were vaccinated with two doses, 21 days apart. Comparison between groups by Mann-Whitney test, bold indicates statistical significance

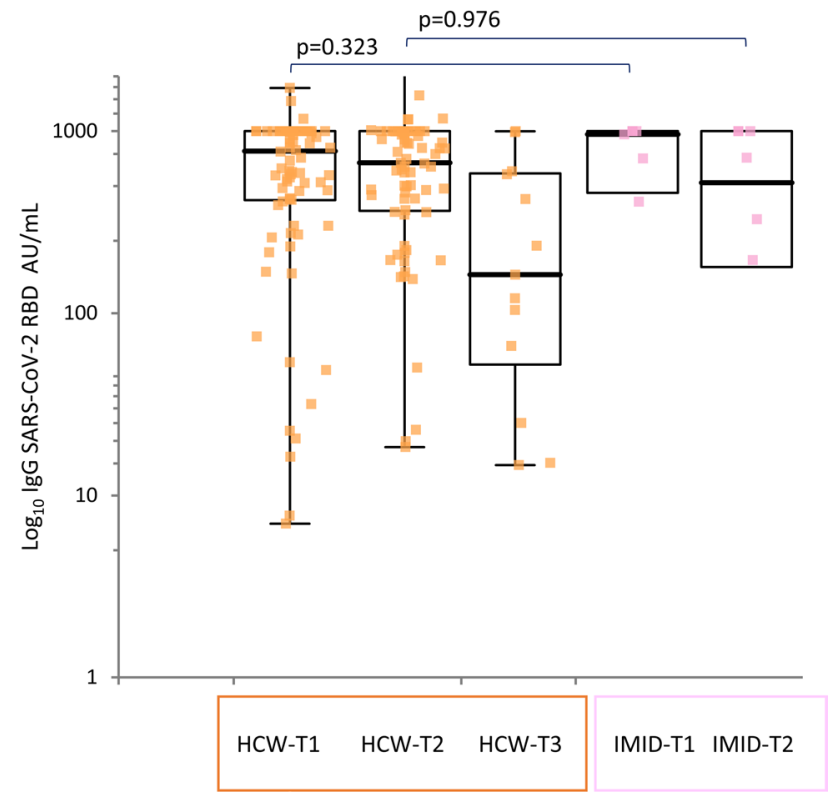

Fig. 2 SARS-CoV-2 anti-S-RBD IgG immune responses in patients and control subjects with previous SARS-CoV-2. IgG titer AntiS-RBD IgG (AU/L) elicited by BNT162b2 at T1 (booster dose), T2 (28 days after booster) and T3 (151 days after first dose) among healthy healthcare workers (HCW) and IMID subjects with previous SARS-CoV-2 infection. All subjects were vaccinated with two doses, 21 days apart. Comparison between groups by Mann-Whitney test, bold indicates statistical significance 


\section{Immunogenicity in the group of naïve IMID patients treated with anti-CD20 drugs}

At T2, 2/7 (28.6\%) subjects were non-responders in the IMID subjects previously treated with B-cell depleting agents (rituximab or ocrelizumab) after two doses of BNT162b2. They were non-responders also at $\mathrm{T} 1$. The median time from last drug infusion until vaccination was 5.5 months (IQR 5.5-6). The median IgG anti-S-RBD levels in this group were significantly lower than other patients with IMID naive to anti-CD20 at both T1 $(p=0.018)$ and T2 $(p=0.011)$ (supplementary materials, Table S3).

\section{Immunogenicity of BNT162B2 in IMID and controls with previous SARS-CoV-2 infection}

In the HCW group $100 \%(111 / 111)$ developed a positive antibody response (defined as $>1 \mathrm{AU} / \mathrm{ml}$ or higher) and an increase in IgG titer at T1 as compared with $88.8 \%(8 / 9)$ of responders at both $\mathrm{T} 1$ and $\mathrm{T} 2$ in the IMID group (Fig. 2 ).

At T1, the median IgG anti-S-RBD levels in the IMID group were 980.2 AU/mL (IQR, 632-1000), then 714.9 AU/ $\mathrm{mL}$ (IQR, 58.31-292.35) at T2.

In controls, at $\mathrm{T} 1$ the median $\mathrm{IgG}$ anti-S-RBD levels were 778.2 AU/mL (IQR, 416-1000), 670 AU/mL (IQR, 363.15-1000) at T2 and then decreasing to $162 \mathrm{AU} / \mathrm{mL}$ (IQR, 45.44-590) at $\mathrm{T} 3$.

The median IgG anti-S-RBD levels in the IMID group with previous SARS-CoV-2 were not significantly different than $\mathrm{HCW}$ group at T1 $(p=0.323)$ and T2 $(p=0.976)$.

\section{Tolerability of BNT162b2 in patients with IMID}

In general, vaccination was well tolerated in all patients and controls. No relapse or overshooting inflammatory response to vaccination was observed in patients with IMID. We detected no relevant safety issues in the enrolled subjects of both groups. Side effects were generally more frequent after the booster dose, and pain at the injection site was most frequently observed in both groups without statistically significant differences between IMID and HCW (both naïve and non-naïve) and among IMID subgroups. Systemic side effects (injection site reaction, headache, chills and arthralgia) were less frequent in patients than in controls $(23.2 \%$ vs. $40 \%$, this finding being significant among naïve IMID ( $p=0.04$, no differences between disease subgroups) and associated with ongoing glucocorticoids $(p=0.032)$.

\section{Discussion}

The ongoing COVID-19 pandemic is being limited also by an unprecedented vaccine program with the innovative use of mRNA vaccines [8]. Due to limitations in the mRNA vaccine trials and to prioritization given to IMID patients for vaccination data about response to COVID-19 mRNA vaccines are of utmost importance to provide data about immunogenicity in this population and to inform health policies. This study shows that BNT162b2, based on mRNA technology, is highly immunogenic in IMID patients as the vast majority (94\%) are responders (defined by development of $\mathrm{IgG}$ to $\mathrm{S}-\mathrm{RBD} \geq 1 \mathrm{AU} / \mathrm{ml}$ ) one month after the second dose. In the subgroup of patients under B-cell depleting agents, this proportion is reduced to about $30 \%$. Among naïve subjects, IMID patients showed a significant difference in the levels of anti-S-RBD IgG than $\mathrm{HCW}$ ( $-33 \%$ at $\mathrm{T} 2$, after the full vaccination course), whereas no significant difference was observed at T3, 5 months after vaccination.

Initial immunogenicity data available for at-risk subgroups showed a high proportion of non-responders and low antibody responses to the SARS-CoV-2 spike protein in patients who received solid-organ transplantation [18], patients treated for solid cancer [19], or in patients with ongoing hemodialysis [20]. In contrast, the available studies to date show that a full course of mRNA vaccine in patients with IMID elicit a response in $78-100 \%$ of subjects [21], showing reduced serum antibody IgG titers and serum neutralizing activity as compared to healthy controls [10, 13, 14, 22-24]. Reduced humoral and cellular immune response $(\mathrm{CD} 8+)$ to COVID-19 mRNA vaccines were found by Haberman et al. in IMID treated with methotrexate and by Mahil et al. in psoriasis $(47 \%$ and $62 \%$ responders, respectively) [23, 25], although this finding has not been confirmed in our and other studies (>80\% responders) $[14,21,22,26]$. Some factors such as the characteristics of enrolled populations and selection criteria may explain this difference.

Even with limitations due to different assays and thresholds used across the available studies, the vaccine-elicited responses are largely indicative of a considerable immunogenicity. The results of the present study are in-line with those findings, and highlight that IMID treatments including GC, DMARDs, biologicals different from B-cell depleting agents could maintain $\mathrm{IgG}$ anti-S-RBD response until 5 months.

Of note, a strong correlation exists between serum neutralizing activity to SARS-COV-2 using PRNT $_{50}$ and anti-S-RBD titer evaluated by the assay used in our study [16]. The median anti-S-RBD found in this study in IMID, respectively, 146.39 $\mathrm{AU} / \mathrm{mL}$ and $60.65 \mathrm{AU} / \mathrm{ml}$ at $\mathrm{T} 2$ and 
T3, may correlate to a high neutralizing titer $(\geq 1: 160$ $\mathrm{PRNT}_{50}$ ) for the majority of patients, and almost all those with $>50 \mathrm{AU} / \mathrm{ml}$ may have neutralizing titers of 1:40-1:80 up to 5 months after vaccination. This is in-line with predictive models of protection from COVID-19 infection and severe disease based on different COVID-19 vaccine trials [27]. Besides IMID, healthy subjects may show low humoral (spike antibody titer or neutralizing antibodies) or cellular response to vaccines. Anti-spike antibody titer (and the related neutralizing activity) declines over months in most vaccinated subjects $[28,29]$, with different rate between subjects previously positive and those naïve to SARS-CoV-2. However, our data do not show significant differences at 5 months between IMID and HCW. The main difference might be in the peak $\operatorname{IgG}$ titer reached after vaccine booster, then this difference decreases over a few months due to a faster decrease in healthy subjects. ( $-79 \%$ than $\mathrm{T} 2$ peak among $\mathrm{HCW}$ vs. $-59 \%$ among IMID). We are not aware of data showing a different efficacy (excess of moderate/severe illness or symptomatic disease) in the first 5-6 months after mRNA vaccination with two doses in IMID versus healthy subjects [30].

Age had an influence at T1 and T2, with a stronger negative correlation in IMID patients than HCW, but not at T3. Previous reports also found that the age impact was timerelated [28, 31], and may be linked to an $\operatorname{IgG}$ response that is faster and of higher magnitude in younger subjects for a short period after the two vaccine doses also for IMID.

In IMID patients, with ongoing immunosuppressive regimens also based on drug combinations and no drug suspension before or during the vaccination schedule, we found no differences in rates of responders neither in anti-S-RBD titer and this may be useful to study the most appropriate vaccination strategies for these patients. Simpler vaccination schedules may increase adherence of patients. The magnitude of response impairment to mRNA COVID-19 vaccine in patients with ongoing B-cells depleting therapies suggest that precise strategies of vaccination should be implemented in the next future [11, 14, 17]. Strategies to rescue the immunogenicity in these patients by repeating a full vaccine course [32] or giving a third dose [33] might be explored in larger studies. Peripheral lymphocytes subpopulations (especially CD19+ counts $>27$ cells $/ \mu \mathrm{L}$ ) are predictive of vaccine response and there is a short period observed from vaccine to infection $[34,35]$, that may indicate the importance of detecting primary non-responders. These findings preliminary suggest that patients treated with anti-CD20 may deserve attention on B-cell reconstitution and/or antibody testing and strict adherence to personal protective measures.

We also assessed humoral responses of nine IMID individuals receiving immunosuppression who had previous natural SARS-CoV-2 infection and received two vaccine doses. There was evidence of robust IgG anti-RBD response in all but one patient, and the humoral response exceeded that of our SARS-CoV-2-naive participants and was similar to that of $\mathrm{HCW}$ with previous infection. The non-responder subject underwent her last rituximab course and COVID19 , respectively, 14 and 12 months before vaccination and peripheral B-cell depletion persisted at time of vaccination. Similar findings have been reported [22, 23].

Systemic side effects after vaccination were less frequent in IMID than in controls as in many previous studies [13, 14, 23], this finding being significantly associated with current treatment with glucocorticoids, but the impact of other factors and biases should be better addressed.

The study limitations are the number of recruited subjects in some subgroups and the absence of formal studies with neutralization assays or cellular immunity, as well as the absence of adenoviral-vector-based vaccinated subjects.

This data highlight the immunogenicity and importance of COVID-19 vaccination in patients with IMID as a measure to contrast pandemics, taking into consideration specific groups such as those treated with B-cell depleting agents. Epidemiological and efficacy studies for special groups are needed to understand whether there is a different immune response kinetics to mRNA vaccination, differentiate the risk for specific subgroups in order to tailor vaccination campaign and determine if further vaccine doses are warranted and their optimal timing.

Supplementary Information The online version contains supplementary material available at https://doi.org/10.1007/s10238-021-00771-3.

Author contributions Study conception and design contributed to DF, AP, LC and MC; Cared for patients, extracted the clinical and lab data contributed to DF, SDG, MC, FM, FC, FS, MS and GU; Acquisition of data contributed to MM, GC and RC; Analysis and interpretation of data contributed to DF, GF, MGC and LC; Drafting of manuscript contributed to DF, RL, GO, AP and LC; Critical revision contributed to SDG, RC, FC, RL and GF. All authors read and worked on the manuscript.

Funding This research was supported by a grant \#2020.2197 of Fondazione di Sardegna, Fondo Coesione e Sviluppo, Regione Autonoma della Sardegna (\# 9417) and by Associazione per l'Avanzamento della Ricerca per i Trapianti O.D.V.

Data availability Data and materials are available from corresponding authors on reasonable request.

\section{Declarations}

Conflict of interest The authors have declared no conflict of interests.

Consent to participate Written consent to participate was obtained for enrolled subjects.

Ethical approval AOU IRB approval number GT/2020/10894 and its extension approved Jan 27th, 2021. 
Open Access This article is licensed under a Creative Commons Attribution 4.0 International License, which permits use, sharing, adaptation, distribution and reproduction in any medium or format, as long as you give appropriate credit to the original author(s) and the source, provide a link to the Creative Commons licence, and indicate if changes were made. The images or other third party material in this article are included in the article's Creative Commons licence, unless indicated otherwise in a credit line to the material. If material is not included in the article's Creative Commons licence and your intended use is not permitted by statutory regulation or exceeds the permitted use, you will need to obtain permission directly from the copyright holder. To view a copy of this licence, visit http://creativecommons.org/licenses/by/4.0/.

\section{References}

1. Saad-Roy CM, Wagner CE, Baker RE, et al. Immune life history, vaccination, and the dynamics of SARS-CoV-2 over the next 5 years. Science. 2020;370:811-8. https://doi.org/10.1126/science. abd7343.

2. Kissler SM, Tedijanto C, Goldstein E, Grad YH, Lipsitch M. Projecting the transmission dynamics of SARS-CoV-2 through the postpandemic period. Science. 2020;368:860-8. https://doi.org/ 10.1126/science.abb5793.

3. Bohn MK, Loh TP, Wang CB, et al. IFCC interim guidelines on serological testing of antibodies against SARS-CoV-2. Clin Chem Lab Med. 2020;58:2001-8. https://doi.org/10.1515/ cclm-2020-1413.

4. Sahin U, Muik A, Derhovanessian E, et al. COVID-19 vaccine BNT162b1 elicits human antibody and TH1 T cell responses. Nature. 2020;586:594-9. https://doi.org/10.1038/ s41586-020-2814-7.

5. Williams JP, Meyers JA. Immune-mediated inflammatory disorders (I.M.I.D.s): the economic and clinical costs. Am J Manag Care. 2002;8:S664-81; quiz S82-5

6. Furer V, Rondaan C, Heijstek MW, et al. 2019 update of EULAR recommendations for vaccination in adult patients with autoimmune inflammatory rheumatic diseases. Ann Rheum Dis. 2020;79:39-52. https://doi.org/10.1136/annrh eumdis-2019-215882.

7. Gluck T, Kiefmann B, Grohmann M, Falk W, Straub RH, Scholmerich J. Immune status and risk for infection in patients receiving chronic immunosuppressive therapy. J Rheumatol. 2005;32:1473-80.

8. Fagni F, Simon D, Tascilar K, et al. COVID-19 and immunemediated inflammatory diseases: effect of disease and treatment on COVID-19 outcomes and vaccine responses. Lancet Rheumatol. 2021;3:e724-36. https://doi.org/10.1016/S2665-9913(21) 00247-2.

9. Curtis JR, Johnson SR, Anthony DD, et al. American college of rheumatology guidance for COVID-19 vaccination in patients with rheumatic and musculoskeletal diseases: version 2 . Arthritis Rheumatol. 2021. https://doi.org/10.1002/art.41877.

10. Geisen UM, Berner DK, Tran F, et al. Immunogenicity and safety of anti-SARS-CoV-2 mRNA vaccines in patients with chronic inflammatory conditions and immunosuppressive therapy in a monocentric cohort. Ann Rheum Dis. 2021. https://doi.org/10. 1136/annrheumdis-2021-220272.

11. Achiron A, Mandel M, Dreyer-Alster S, et al. Humoral immune response to COVID-19 mRNA vaccine in patients with multiple sclerosis treated with high-efficacy disease-modifying therapies. Ther Adv Neurol Disord. 2021;14:17562864211012836. https:// doi.org/10.1177/17562864211012835.

12. Haberman R, Axelrad J, Chen A, et al. Covid-19 in immunemediated inflammatory diseases-case series from New York.
N Engl J Med. 2020;383:85-8. https://doi.org/10.1056/NEJMc 2009567.

13. Simon D, Tascilar K, Fagni F, et al. SARS-CoV-2 vaccination responses in untreated, conventionally treated and anticytokine-treated patients with immune-mediated inflammatory diseases. Ann Rheum Dis. 2021. https://doi.org/10.1136/annrh eumdis-2021-220461.

14. Furer V, Eviatar T, Zisman D, et al. Immunogenicity and safety of the BNT162b2 mRNA COVID-19 vaccine in adult patients with autoimmune inflammatory rheumatic diseases and in the general population: a multicentre study. Ann Rheum Dis. 2021. https:// doi.org/10.1136/annrheumdis-2021-220647.

15. Walsh EE, Frenck RW Jr, Falsey AR, et al. Safety and immunogenicity of two RNA-based covid-19 vaccine candidates. N Engl J Med. 2020;383:2439-50. https://doi.org/10.1056/NEJMo a2027906

16. Padoan A, Bonfante F, Cosma C, et al. Analytical and clinical performances of a SARS-CoV-2 S-RBD IgG assay: comparison with neutralization titers. Clin Chem Lab Med. 2021;59:144452. https://doi.org/10.1515/cclm-2021-0313.

17. Simon D, Tascilar K, Schmidt K, et al. Brief report: humoral and cellular immune responses to SARS-CoV-2 infection and vaccination in B cell depleted autoimmune patients. Arthritis Rheumatol. 2021. https://doi.org/10.1002/art.41914.

18. Rozen-Zvi B, Yahav D, Agur T, et al. Antibody response to SARS-CoV-2 mRNA vaccine among kidney transplant recipients: a prospective cohort study. Clin Microbiol Infect. 2021. https://doi.org/10.1016/j.cmi.2021.04.028.

19. Barriere J, Chamorey E, Adjtoutah Z, et al. Impaired immunogenicity of BNT162b2 anti-SARS-CoV-2 vaccine in patients treated for solid tumors. Ann Oncol. 2021. https://doi.org/10. 1016/j.annonc.2021.04.019.

20. Grupper A, Sharon N, Finn T, et al. Humoral response to the Pfizer BNT162b2 vaccine in patients undergoing maintenance hemodialysis. Clin J Am Soc Nephrol. 2021. https://doi.org/10. 2215/CJN.03500321.

21. Jena A, Mishra S, Deepak P, et al. Response to SARS-CoV-2 vaccination in immune mediated inflammatory diseases: systematic review and meta-analysis. Autoimmun Rev. 2021. https://doi.org/10.1016/j.autrev.2021.102927.

22. Kennedy NA, Lin S, Goodhand JR, et al. Infliximab is associated with attenuated immunogenicity to BNT162b2 and ChAdOx1 nCoV-19 SARS-CoV-2 vaccines in patients with IBD. Gut. 2021. https://doi.org/10.1136/gutjnl-2021-324789.

23. Mahil SK, Bechman K, Raharja A, et al. The effect of methotrexate and targeted immunosuppression on humoral and cellular immune responses to the COVID-19 vaccine BNT162b2: a cohort study. Lancet Rheumatol. 2021. https://doi.org/10.1016/ S2665-9913(21)00212-5.

24. Valor-Mendez L, Tascilar K, Simon D, et al. Correspondence on "Immunogenicity and safety of anti-SARS-CoV-2 mRNA vaccines in patients with chronic inflammatory conditions and immunosuppressive therapy in a monocentric cohort.” Ann Rheum Dis. 2021. https://doi.org/10.1136/annrh eumdis-2021-220898.

25. Haberman RH, Herati R, Simon D, et al. Methotrexate hampers immunogenicity to BNT162b2 mRNA COVID-19 vaccine in immune-mediated inflammatory disease. Ann Rheum Dis. 2021. https://doi.org/10.1136/annrheumdis-2021-220597.

26. Deepak P, Kim W, Paley MA, et al. Effect of immunosuppression on the immunogenicity of mRNA vaccines to SARS-CoV-2: a prospective cohort study. Ann Intern Med. 2021. https://doi.org/ 10.7326/M21-1757.

27. Khoury DS, Cromer D, Reynaldi A, et al. Neutralizing antibody levels are highly predictive of immune protection from 
symptomatic SARS-CoV-2 infection. Nat Med. 2021. https://doi. org/10.1038/s41591-021-01377-8.

28. Naaber P, Tserel L, Kangro K, et al. Dynamics of antibody response to BNT162b2 vaccine after six months: a longitudinal prospective study. Lancet Reg Health Eur. 2021. https://doi.org/ 10.1016/j.lanepe.2021.100208.

29. Favresse J, Bayart JL, Mullier F, et al. Antibody titres decline 3-month post-vaccination with BNT162b2. Emerg Microbes Infect. 2021;10:1495-8. https://doi.org/10.1080/22221751.2021. 1953403.

30. Bergwerk M, Gonen T, Lustig Y, et al. Covid-19 breakthrough infections in vaccinated health care workers. N Engl J Med. 2021. https://doi.org/10.1056/NEJMoa2109072.

31. Bayart JL, Morimont L, Closset M, et al. Confounding factors influencing the kinetics and magnitude of serological response following administration of BNT162b2. Microorganisms. 2021. https://doi.org/10.3390/microorganisms9061340.

32. Albach FN, Burmester GR, Biesen R. Successful BNT162b2 booster vaccinations in a patient with rheumatoid arthritis and initially negative antibody response. Ann Rheum Dis. 2021. https:// doi.org/10.1136/annrheumdis-2021-220834.
33. Kamar N, Abravanel F, Marion O, Couat C, Izopet J, Del Bello A. Three doses of an mRNA covid-19 vaccine in solid-organ transplant recipients. N Engl J Med. 2021. https://doi.org/10.1056/ NEJMc2108861.

34. Moor MB, Suter-Riniker F, Horn MP, et al. Humoral and cellular responses to mRNA vaccines against SARS-CoV-2 in patients with a history of CD20 B-cell-depleting therapy (RituxiVac): an investigator-initiated, single-centre, open-label study. Lancet Rheumatol. 2021. https://doi.org/10.1016/s2665-9913(21) 00251-4.

35. Lawson-Tovey S, Hyrich KL, Gossec L, et al. SARS-CoV-2 infection after vaccination in patients with inflammatory rheumatic and musculoskeletal diseases. Ann Rheum Dis. 2021. https://doi.org/ 10.1136/annrheumdis-2021-221217.

Publisher's Note Springer Nature remains neutral with regard to jurisdictional claims in published maps and institutional affiliations. 